Canadian

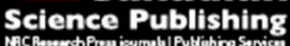

Applied Physiology, Nutrition, and Metabolism Physiologie appliquée, nutrition et métabolisme

\title{
Impact of Intradialytic Exercise Intensity on Urea Clearance in Hemodialysis Patients
}

\begin{tabular}{|c|c|}
\hline Journal: & Applied Physiology, Nutrition, and Metabolism \\
\hline Manuscript ID & apnm-2017-0460.R1 \\
\hline Manuscript Type: & Brief communication \\
\hline Date Submitted by the Author: & 12-Sep-2017 \\
\hline Complete List of Authors: & $\begin{array}{l}\text { Brown, Paul; University of Calgary Faculty of Kinesiology, } \\
\text { Rowed, Kylie; University of Calgary Faculty of Kinesiology } \\
\text { Shearer, Jane; University of Calgary, } \\
\text { MacRae, Jennifer; University of Calgary, Cumming School of Medicine } \\
\text { Parker, Kristen; Southern Alberta Renal Program, }\end{array}$ \\
\hline \multicolumn{2}{|l|}{$\begin{array}{r}\text { Is the invited manuscript for } \\
\text { consideration in a Special } \\
\text { Issue? : }\end{array}$} \\
\hline Keyword: & $\begin{array}{l}\text { exercise intensity }<\text { exercise, end stage kidney disease, hemodialysis, urea } \\
\text { clearance, heart rate }\end{array}$ \\
\hline
\end{tabular}




\title{
Brief Communication
}

\section{Impact of Intradialytic Exercise Intensity on Urea Clearance in Hemodialysis Patients}

*Paul David Stuart Brown ${ }^{1}$, Kylie Rowed ${ }^{1}$, Jane Shearer $^{1,2}$, Jennifer M. MacRae ${ }^{3}$, Kristen Parker ${ }^{3,4}$

${ }^{1}$ Faculty of Kinesiology, University of Calgary. Calgary, Alberta, Canada. ${ }^{2}$ Department of Biochemistry and Molecular Biology, Cumming School of Medicine. University of Calgary. Calgary, Alberta, Canada. ${ }^{3}$ Department of Nephrology. Cumming School of Medicine, Calgary, Alberta, Canada. ${ }^{4}$ Southern Alberta Renal Program, Alberta Health Services, Calgary, Alberta. Canada.

Disclosure: The authors declare no conflicts of interest.

\begin{abstract}
: 72 words
\end{abstract}
Main Text: 2998 words

*Corresponding Author: Paul Brown. Faculty of Kinesiology. 2500 University Drive NW. University of Calgary. Calgary, Alberta, Canada. T2N 1N4. Email: pdbrown@ucalgary.ca. Tel: (250) 341-7365 


\begin{abstract}
Intradialytic exercise (IDE) has been shown to benefit dialysis efficacy; however, the effect of IDE intensity is unknown. Dialyzer urea clearance ( $\mathrm{K}$ urea, $\mathrm{ml} / \mathrm{min}$ ) was significantly greater during both IDE protocols (55\% and 70\% maximal heart rate, HRmax), compared to no IDE ( $\mathrm{p}<0.05)$. No significant difference in K urea was found between IDE protocols (55\% vs. 70\% HRmax) (p>0.05). Results show that higher intensity IDE has no additional benefit on $\mathrm{K}$ urea.
\end{abstract}

Key words: exercise; intensity; end-stage kidney disease; hemodialysis; urea; heart rate 


\section{INTRODUCTION}

Kidney failure rates have tripled in the past 20 years, resulting in an estimated 41,931 Canadians being treated for the disease (CORR Report 2015). Hemodialysis (HD) provided within an institutional setting (e.g. hospital) is the most common treatment for kidney failure, costing the Canadian health care system $\$ 95,000$ - \$107,000 per patient per year (CORR Report 2015). Intradialytic exercise (IDE) as a therapy for HD patients is utilized in few North American dialysis programs. Benefits of IDE for patients include improvements in aerobic capacity $\left(\dot{\mathrm{VO}}_{2}\right.$ peak)(Ouzouni et al. 2009), leg muscle strength (Chen et al. 2010), quality of life (Ouzouni et al. 2009), as well as lower markers of inflammation (Afshar et al. 2010), and hospital usage (Parker et al. 2015).

Another important benefit of IDE is enhanced solute removal and efficacy of the dialysis treatment itself (Kong et al. 1999, Parsons et al. 2006). Compared to dialysis at rest, IDE increases blood flow and perfusion of the exercising musculature, thereby increasing the surface area of exchange between the blood and the muscle tissue. Subsequently, more waste products (e.g. urea) can diffuse from the muscle into circulation — to be removed via dialysis — during IDE compared to dialysis at rest.

The effectiveness of IDE is shown in measures of dialysis efficacy, such as $K t / V$, where $K$ is dialyzer clearance of urea in $\mathrm{ml} / \mathrm{min}, \mathrm{t}$ is the dialysis time in minutes, and $\mathrm{V}$ is the volume of distribution of urea in $\mathrm{ml}$ (roughly the patient's total body water) (Parsons et al. 2006, Giannaki et al. 2011). Dialyzer clearance of urea $(\mathrm{K}$ urea, $\mathrm{ml} / \mathrm{min})$ is another common measure of dialysis efficacy as values are often reported in "real time" by dialysis machines. While IDE is beneficial, the impact of exercise intensity of $\mathrm{K}$ urea and other indices of dialysis are not known. Therefore, the purpose of the present study was to determine whether increasing IDE intensity enhances dialysis efficacy in a HD population. This is an important question as dialysis efficacy likely impacts patient morbidity and mortality (Greene et al. 2005). 


\section{METHODS}

Subjects and recruitment. This clinical cross-over design study was approved by the Conjoint Health Ethics Research Board of the University of Calgary. Patients who were dialyzing thrice weekly and participating in the IDE program at the onset of the study were recruited from two Calgary outpatient HD units from January 2015 to November 2016. After meeting these inclusion criteria, 22 patients signed informed consent. Researchers excluded those with a poorly functioning access, myocardial infarction within the last six months, severe aortic valve stenosis, decompensated congestive heart failure, unstable angina, pacemakers, and beta-blocker usage.

Hemodialysis and monitoring. HD was conducted on a Fresenius 5008 machine (Fresenius Medical Care, Bad Homburg, Germany) is equipped with Online Clearance Monitoring (OCM), which is an efficient method for determining solute clearance. Pump speed, dialysate flow, dialysis time, and participants' dry weight had to remain constant throughout all three protocols to be used for analysis. The majority of patients (15/17) used a constant sodium profile throughout dialysis. If the participant dialyzed with a central venous catheter $(\mathrm{CVC})$, researchers ensured that there were no differences in arterial and venous line connection. Subjects underwent HD for one hour prior to initiating each protocol (control or exercise). To access the impact of exercise on urea kinetics, $\mathrm{K}$ urea (ml/min), peak urea clearance $(\mathrm{ml} / \mathrm{min})$, calculated $\mathrm{Kt} / \mathrm{V}$, and the percent change in urea clearance from baseline was assessed during each session.

Exercise protocol. A schematic of the experimental design and exercise protocol is shown in Figure 1 A. Participants were randomized to three different protocols during the mid-week HD treatment over three consecutive weeks (1/week). The three protocols were 1) no exercise, 2) 30-minutes of exercise at 55\% of age-predicted maximal heart rate (HRmax), 3) 30-minutes of exercise at 70\% HRmax. On exercise days, participants started their exercise after $60 \mathrm{~min}$ of $\mathrm{HD}$, in order to collect baseline data. At one hour 
of HD, a kinesiologist placed a Monark Rehab Trainer 881E (Monark Exercise AB, Vansbro, Sweden), set to the lowest resistance, at the base of the dialysis chair.

Each participant was then shown a modified 0-10 point rating of perceived exertion (RPE) scale (Borg 1970). Participants self-selected an exercise intensity that fit their perception of "easy", or two out of 10 on the Borg scale (Borg 1970), on the 55\% HRmax day. Participants self-selected an exercise intensity that fit their perception of "somewhat hard", or four out of 10 on the Borg scale (Borg 1970), on the $70 \%$ HRmax day. In addition, the patient was encouraged to train as close to a HR at their percentage of HRmax (220-age in years; then $55 \%$ and $70 \%$ of max value). Pre-exercise values (i.e. blood pressure, HR, and oxygen saturation) were recorded immediately before IDE, while mid-exercise values were recorded every 10 minutes. Post-exercise values were recorded two minutes after IDE cessation. The research team monitored changes to K urea by recording new values (as they appeared on the HD machine's OCM diagram) throughout the duration of the dialysis treatment $(\sim 10$ minute intervals). The percent change between the last pre-exercise $\mathrm{K}$ urea value and the peak of the highest $\mathrm{K}$ urea value achieved during exercise was calculated.

Statistical Analysis. Statistical analysis was performed using GraphPad Prism (Version 7.0 La Jolla, CA). Differences between treatments were determined by a one way repeated analysis of variance (ANOVA), followed by a Tukey's post-hoc test where $\mathrm{p}<0.05$ was considered to be significant. Data are expressed as mean $\pm \mathrm{SEM}$.

\section{RESULTS}

In total, 22 participants provided informed consent and participated in the study. After starting the study, three participants were unable to complete the protocols because of CVC variability between runs, and a final participant could not perform the exercise following a leg injury from a fall. Participant demographics are outlined in Table 1. 
$\mathrm{Kt} / \mathrm{V}$ was slightly, but significantly greater during the $55 \%$ session compared to the control session (Figure 1B, $\mathbf{p}<\mathbf{0 . 0 5}$ ). However, there was no difference in $\mathrm{Kt} / \mathrm{V}$ between the $70 \%$ session and the control session (Figure 1B, $\mathbf{p}>\mathbf{0 . 0 5}$ ). No pre-exercise differences in K urea were found between treatment groups $(\mathrm{p}>0.05)$, but $\mathrm{K}$ urea was found to be significantly greater during exercise in both IDE sessions (55\% and 70\%) compared to the control session (Figure 1C, $\mathbf{p}<\mathbf{0 . 0 5}$ ). Unexpectedly, there were no differences between $\mathrm{K}$ urea with exercise intensity (e.g. 55\% vs. 70\%) (p>0.05). Following exercise, urea clearance returned to baseline levels on all groups with no difference between treatments $(p<0.05)$. Peak K urea and the percentage change in clearance was found to be significantly greater during both IDE sessions compared to the control session $(\mathbf{p}<\mathbf{0 . 0 5}$, Figure 1D, 1E), with no differences noted between IDE intensities ( $\mathrm{p}>0.05)$.

Two indices were used to gauge exercise intensity in the present study, the Borg (RPE) and HR. HR was significantly greater at all three time points (i.e. 10, 20, and 30 minutes) from the 55\% to the $70 \%$ trial, with an average increase of $7.3 \pm 1.3 \mathrm{bpm}$ between the $55 \%$ and $70 \%$ treatment groups $(\mathrm{p}<0.05)($ Figure 1F). This increase in physiological exertion from the $55 \%$ to the $70 \%$ protocol was consistent with the participant RPE results shown in Figure 1G that was greater at all three time points assessed. No adverse events as a result of the exercise were observed although two subjects did report "burning/pain in his lower leg" and feeling "a bit dizzy" upon completion of the 70\% IDE session.

\section{DISCUSSION}

To our knowledge, this is the first study to examine the effect of IDE intensity on real time K urea and other dialysis indices. A significant increase in K urea was observed during IDE compared to dialysis at rest $(5.5 \pm 1.9 \%$ and $12.4 \pm 2.6 \%$ increase for 55 and $70 \%$, respectively). However, no difference in $\mathrm{K}$ urea between the $55 \%$ and the $70 \%$ protocol was observed. One interpretation of this finding is that greater IDE intensity may not induce greater $\mathrm{K}$ urea. The implication of this is that HD patients may not have to work as hard during IDE to achieve an increase in K urea, compared to dialysis 
at rest. An alternate interpretation is that HD patients were unable to achieve a significant enough increase in exercise intensity with the $70 \%$ protocol due to their advanced age ( $>66$ years), significant comorbidities $(>2)$, and disease burden ( $>5$ years on dialysis).

Despite these findings, it must be emphasized that there are other potential benefits to exercising at a greater intensity. For example, Painter et al. (2002) observed a significant increase in $\dot{\mathrm{VO}}_{2}$ peak when incorporating a 30-minute, 70\%+ HRmax IDE protocol for 5 months. Konstantinidou et al. (2002) also observed an increase in $\dot{\mathrm{VO}}_{2}$ peak, as well as anaerobic threshold, exercise time, and maximal ventilation when incorporating a 60-minute, 70\% HRmax IDE protocol for 6 months. In addition to cardiorespiratory benefits of higher intensity IDE, improvements in muscular strength, psychological status and quality of life have been reported (Konstantinidou et al. 2002). However, it should be noted improvements with greater intensity exercise occurred over a longer duration in these studies (5-6 months) as compared to the single dialysis run in the present study.

The impact of IDE on Kt/V is inconsistent in the literature, with both positive (Kong et al. 1999, Parsons et al. 2006) and no changes reported (Afshar et al. 2010, Kirkman et al. 2013). Compared to the control session, $\mathrm{Kt} / \mathrm{V}$ was found to be slightly, but significantly greater during the $55 \%$ protocol, but not during the $70 \%$ protocol. Reasons for a lack of $\mathrm{Kt} / \mathrm{V}$ increase during the $70 \%$ session are not known, but may be due to increased muscle blood flow and greater cardiac output (leading to increased urea recirculation), reduced effective dialysis time due to increased alarm interruptions, or the small sample size.

Two different indices were used to assess exercise intensity in this study including HRmax and RPE. It should be noted that no participants achieved $70 \%$ of their age-predicted HRmax for the total exercise duration (while only nine patients achieved 55\% of their age-predicted HRmax). Reasons for lack of attainment of the predicted HR increase include: lack of sympathetic response due to autonomic neuropathy, and greater perceived exertion at a given HR due to limited leg strength (Konstantinidou et al. 2002, Raj et al. 2008). HD patients tend to be limited by peripheral factors, as opposed to central 
factors, during IDE. This decrease in leg strength is mainly caused by leg muscular atrophy, resulting from protein hypercatabolism in HD patients (Raj et al. 2008). HD is also known to promote plasma amino acid loss, with 10-12 g of amino acids removed in the dialysate per HD treatment resulting in muscle protein hypercatabolism and amino acid release from muscle, resulting in muscle atrophy and decreased muscle strength in HD patients (Raj et al. 2008).

A potential barrier to IDE includes fear of complications from exercise. Two participants in this study reported undesirable symptoms during the $70 \%$ protocol. These symptoms were minor issues, and resolved when the IDE stopped. IDE adverse effects are rare and usually minor in nature. A metaanalysis by Smart et al. (2013) found that no randomized controlled trials involving exercise with chronic renal failure patients have reported a death directly related to exercise in over 30,000 patienthours of exercise.

It should be noted that technique of OCM may have missed an exercise induced increase in K urea if timing of the conductivity measurement occurred after the period of exercise. Thus, a limitation of our study is that the OCM was not routinely determined either at the middle or prior to the end of the IDE session. Another limitation is the use of HR response to define exercise intensity. Determining the intensity is challenging due to the variation in the prediction of the HRmax, especially in this patient population for the reasons outlined above.

In conclusion, an increase in $\mathrm{K}$ urea and $\mathrm{Kt} / \mathrm{V}$ was observed during IDE at 55\% compared to rest. Increasing exercise intensity did not significantly increase either value during IDE and highintensity IDE may not be necessarily better than low-intensity IDE in terms of dialysis efficacy, however, higher-intensity IDE may provide additional benefits. From a practical perspective, lowerintensity IDE may be less intimidating to HD patients compared to high-intensity IDE, and may result in greater compliance. The results of this study suggest that some exercise is better than no exercise for HD efficacy, even if the participant cannot achieve a high IDE intensity. 


\section{ACKNOWLEDGEMENTS}

We wish to acknowledge the contribution of nurses who assisted with data collection. We also wish to thank all of the participants for their involvement in this study. JS is funded by the Natural Sciences and Engineering Research Council of Canada.

\section{Conflict of interest statement}

The authors have no conflicts of interest to declare. 


\section{REFERENCES}

Afshar, R., Shegarfy, L., Shavandi, N., and Sanavi, S. 2010. Effects of aerobic exercise and resistance training on lipid profiles and inflammation status in patients on maintenance hemodialysis. Indian J. Nephrol. 20(4): 185-189. doi:10.4103/0971-4065.73442. PMID:21206679.

Borg, G. 1970. Perceived exertion as an indicator of somatic stress. Scand. J. Rehabil. Med. 2(2): 92-98. PMID: 5523831.

Canadian Organ Replacement Register (CORR) Report. 2015. Treatment of end-stage organ failure in Canada. 2004 to 2013. Canadian Institute for Health Information, Ottawa, Ont., Canada.

Chen, J.L., Godfrey, S., Ng, T.T., Moorthi, R., Liangos, O., Ruthazer, R., Jaber, B.L., Levey, A.S., and Castaneda-Sceppa, C. 2010. Effect of intra-dialytic, low-intensity strength training on functional capacity in adult haemodialysis patients: a randomized pilot trial. Nephrol. Dial. Transplant. 25(6): 1936-1943. doi:10.1093/ndt/gfp739. PMID:20100734.

Giannaki, C.D., Stefanidis, I., Karatzaferi, C., Liakos, N., Roka, V., Ntente, I., and Sakkas, G.K. 2011. The effect of prolonged intradialytic exercise in hemodialysis efficiency indices. Am. Soc. Artif. Intern. Organs 57(3): 213-218. doi:10.1097/MAT.0b013e318215dc9e. PMID:21412149.

Greene, T., Daugirdas, J.T., Depner, T.A., Allon, M., Beck, G., Chumlea, C., Delmez, J., Gotch, F., Kusek, J.W., Levin, N., Owen, W., Schulman, G., Star, R., Toto, R., and Eknoyan, G. 2005. Association of achieved dialysis dose with mortality in the hemodialysis study: an example of “dose-targeting bias”. J. Am. Soc. Nephrol. 16(11): 3371-3380. doi:10.1681/ASN.2005030321. PMID:16192421.

Kirkman, D.L., Roberts, L.D., Kelm, M., Wagner, J., Jibani, M.M., and Macdonald, J.H. 2013. Interaction between intradialytic exercise and hemodialysis adequacy. Am. J. Nephrol. 38(6): 475-482. doi:10.1159/000356340. PMID:24296748. 
Kong, C.H., Tattersall, J.E., Greenwood, R.N., and Farrington, K. 1999. The effect of exercise during haemodialysis on solute removal. Nephrol. Dial. Transplant. 14(12): 2927-2931. doi:10.1093/ndt/14.12.2927. PMID:10570099.

Konstantinidou, E., Koukouvou, G., Kouidi, E., Deligiannis, A., and Tourkantonis, A. 2002. Exercise training in patients with end-stage renal disease on hemodialysis: comparison of three rehabilitation programs. J. Rehabil. Med. 34(1): 40-45. doi:10.1080/165019702317242695. PMID:11900261.

Ouzouni, S., Kouidi, E., Sioulis, A., Grekas, D., and Deligiannis, A. 2009. Effects of intradialytic exercise training on health-related quality of life indices in haemodialysis patients. Clin. Rehabil. 23(1): 53-63. doi:10.1177/0269215508096760. PMID:19114437.

Painter, P., Moore, G., Carlson, L., Paul, S., Myll, J., Phillips, W., and Haskell, W. 2002. Effects of exercise training plus normalization of hematocrit on exercise capacity and health-related quality of life. Am. J. Kidney Dis. 39(2): 257-265. National Kidney Foundation, Inc. doi:10.1053/ajkd.2002.30544. PMID:11840365.

Parker, K., Zhang, X., Lewin, A., and MacRae, J.M. 2015. The association between intradialytic exercise and hospital usage among hemodialysis patients. Appl. Physiol. Nutr. Metab. 40(4): 371-378. doi:10.1139/apnm-2014-0326. PMID:25819883.

Parsons, T.L., Toffelmire, E.B., and King-VanVlack, C.E. 2006. Exercise training during hemodialysis improves dialysis efficacy and physical performance. Arch. Phys. Med. Rehabil. 87(5): 680-687. doi:10.1016/j.apmr.2005.12.044. PMID:16635631.

Raj, D.S., Sun, Y., and Tzamaloukas, A.H. 2008. Hypercatabolism in dialysis patients. Dial. Transplant. 17(6): 589-594. doi:10.1097/MNH.0b013e32830d5bfa. PMID:18941351.

Smart, N., McFarlane, J., and Cornelissen, V. 2013. The effect of exercise therapy on physical function, biochemistry and dialysis adequacy in haemodialysis patients: a systematic review and metaanalysis. Open J. Nephrol. 3(1): 25-36. doi:10.4236/ojneph.2013.31005. 
Table 1. Demographic profile of the intradialytic exercise intensity study group $(n=17$, values reported as mean \pm SEM unless otherwise indicated).

\section{Subject Characteristic}

\begin{tabular}{|c|c|}
\hline Age (yr) & $66.6(2.8)$ \\
\hline Male $(\%)$ & $10(58.8)$ \\
\hline Female $(\%)$ & $7(41.2)$ \\
\hline CVC (\%) & $2(11.8)$ \\
\hline HD vintage (mo) & $65.4(8.2)$ \\
\hline Intradialytic exercise vintage (mo) & $47.7(6.1)$ \\
\hline \multicolumn{2}{|l|}{ Ethnicity (\%) } \\
\hline Caucasian & $11(64.7)$ \\
\hline Asian & $2(11.8)$ \\
\hline Middle Eastern & $2(11.8)$ \\
\hline African American & $1(5.9)$ \\
\hline East Indian & $1(5.9)$ \\
\hline \multicolumn{2}{|l|}{ Primary diagnosis $(\%)$} \\
\hline Diabetes mellitus & $5(29.4)$ \\
\hline GN/Autoimmune & $3(17.6)$ \\
\hline Polycystic kidney disease & $3(17.6)$ \\
\hline Hypertension & $2(11.8)$ \\
\hline Ischemic nephropathy & $2(11.8)$ \\
\hline Obstructive uropathy & $1(5.9)$ \\
\hline \multicolumn{2}{|l|}{ Comorbidity $(\%)$} \\
\hline Hypertension & $9(52.9)$ \\
\hline Diabetes mellitus & $8(47.1)$ \\
\hline $\mathrm{CHF}$ & $4(23.5)$ \\
\hline CAD & $3(17.6)$ \\
\hline CVA/TIA & $3(17.6)$ \\
\hline COPD & $3(17.6)$ \\
\hline Cancer & $3(17.6)$ \\
\hline Angina & $1(5.9)$ \\
\hline MI & $1(5.9)$ \\
\hline \multicolumn{2}{|l|}{ Laboratory values at baseline } \\
\hline Potassium (mmol/L) & $4.6(0.2)$ \\
\hline Hemoglobin $(g / L)$ & $105.9(2.7)$ \\
\hline Calcium $(\mathrm{mmol} / \mathrm{L})$ & $2.2(0.0)$ \\
\hline Phosphorous (mmol/L) & $1.6(0.1)$ \\
\hline Albumin $(\mathrm{g} / \mathrm{L})$ & $33.1(0.6)$ \\
\hline Creatinine $(\mu \mathrm{mol} / \mathrm{L})$ & $650.6(39.2)$ \\
\hline Urea $(\mathrm{mmol} / \mathrm{L})$ & $18.6(1.8)$ \\
\hline $\operatorname{GFR}\left(\mathrm{ml} / \mathrm{min} / 1.73 \mathrm{~m}^{2}\right)$ & $6.8(0.7)$ \\
\hline
\end{tabular}

$\mathrm{CVC}=$ central venous catheter, $\mathrm{HD}=$ hemodialysis, $\mathrm{GN}=$ glomerulonephritis, $\mathrm{CHF}=$ congestive heart failure, $\mathrm{CAD}=$ coronary artery disease, $\mathrm{CVA}=$ cerebrovascular accident, $\mathrm{TIA}=$ transient ischemic attack, $\mathrm{COPD}=$ chronic obstructive pulmonary disease, $\mathrm{MI}=$ myocardial infarction, $\mathrm{GFR}=$ glomerular filtration rate. 


\section{FIGURE LEGEND}

Figure 1. A: Schematic of the experimental protocol. B: Kt/V ratio value obtained from the dialysis run ( $\sim$ h total) for the three experimental conditions (control, $55 \%$, and $70 \%$ intradialytic exercise). C: Urea clearance $(\mathrm{K}, \mathrm{ml} / \mathrm{min})$ values obtained at baseline, during and post exercise in control, $55 \%$ and $70 \%$ intradialytic exercise. An average of three measures per subject, per time period (e.g. baseline) were obtained and averaged for each segment. D: Peak urea clearance $(\mathrm{K}, \mathrm{ml} / \mathrm{min})$ in each of the three conditions of the dialysis period. E: Percent change in urea clearance from baseline. F: Average heart rate (bpm). G: Ratings of perceived exertion (Scale of 0-10, Borg) assessed every 10 minutes during the intradialytic exercise. All data represent $n=17$ subjects and are displayed as mean \pm SEM. * Represents a significant difference from control $(\mathrm{p}<0.05) ; \wedge$ represents a statistically significant difference between $55 \%$ and $70 \%$ intradialytic exercise intensities $(\mathrm{p}<0.05)$. 
A



B

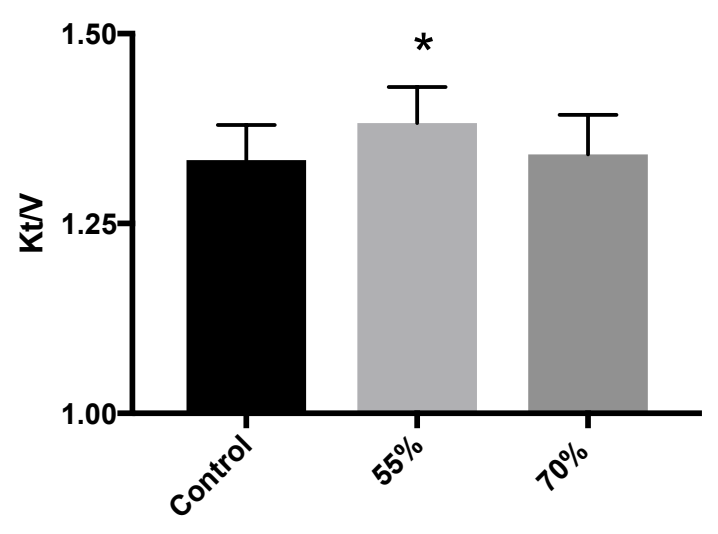

D
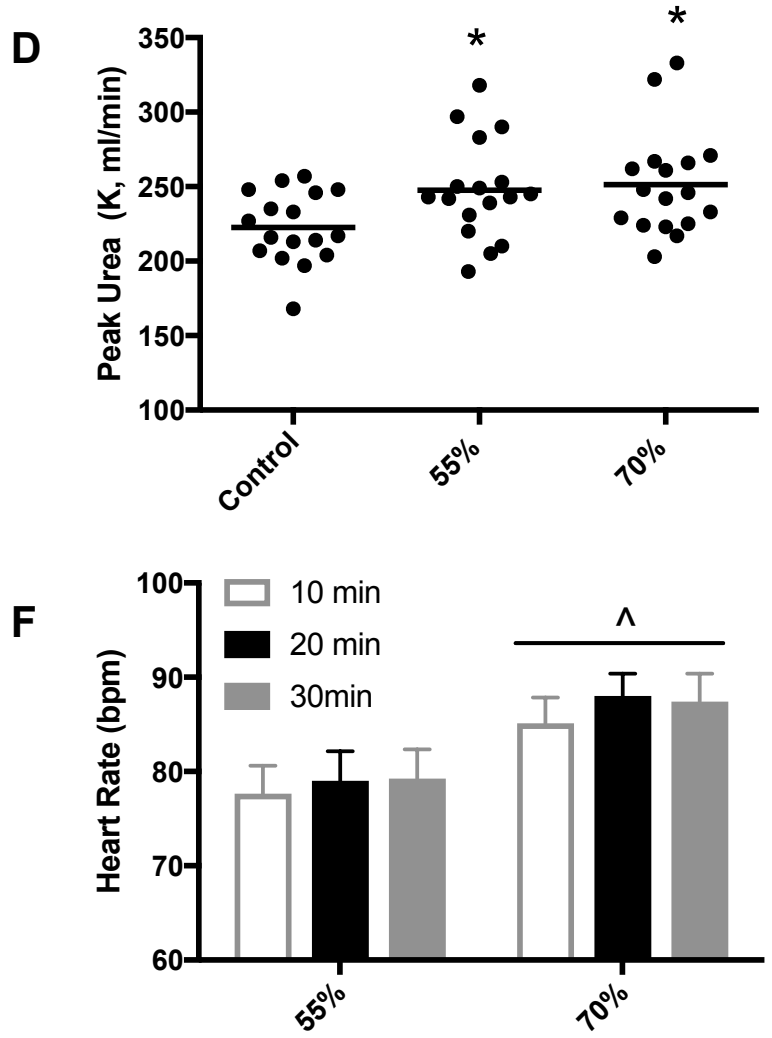

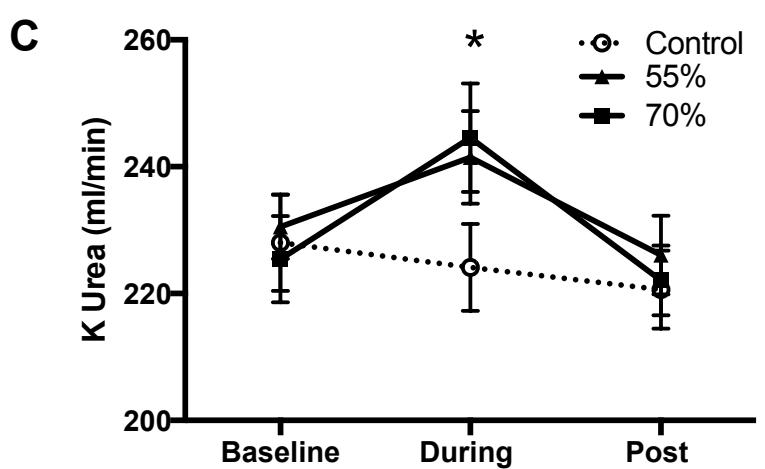

E

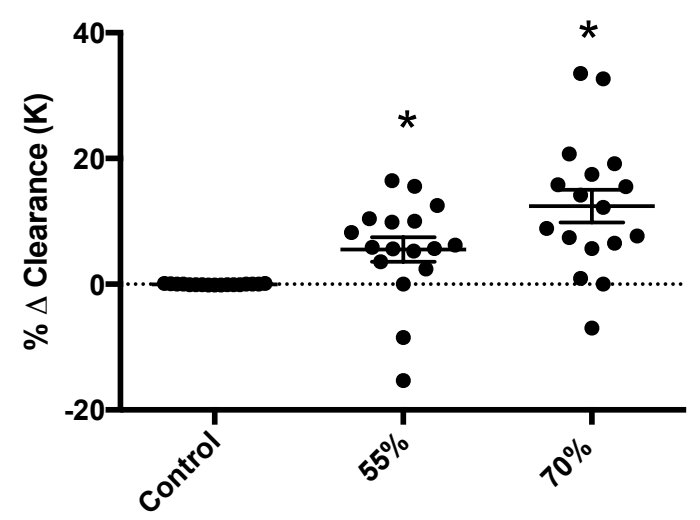

G

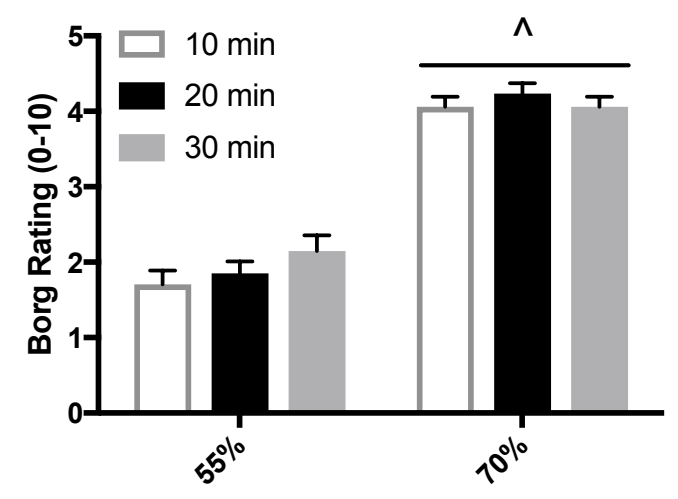

